\title{
Computing Radial Drawings on the Minimum Number of Circles ${ }^{\star}$
}

\author{
Emilio Di Giacomo ${ }^{1}$, Walter Didimo ${ }^{1}$, Giuseppe Liotta $^{1}$, and Henk Meijer ${ }^{2}$ \\ 1 Università degli Studi di Perugia, Perugia, Italy \\ \{digiacomo, didimo, liotta\}@diei.unipg.it \\ 2 School of Computing, Queen's University, Kingston, Ontario, Canada \\ henk@cs . queensu.ca
}

\begin{abstract}
A radial drawing is a representation of a graph in which the vertices lie on concentric circles of finite radius. In this paper we study the problem of computing radial drawings of planar graphs by using the minimum number of concentric circles. We assume that the edges are drawn as straight-line segments and that co-circular vertices can be adjacent. It is proven that the problem can be solved in polynomial time.
\end{abstract}

\section{Introduction}

A radial drawing is a representation of a graph in which the vertices are constrained to lie on concentric circles of finite radius. Drawing graphs radially is relevant in situations where it is important to display a graph with the constraint that some vertices are drawn "more central" than others. Examples of such applications include social networks analysis (visualization of policy networks and co-citation graphs), operating systems (visualization of filesystems), cybergeography (visualization of Web maps and communities), and bioinformatics (visualization of protein-protein interaction diagrams); see e.g. [4, 8, 9].

This paper investigates crossing-free radial drawings of planar graphs. Let $G$ be a planar graph. A crossing-free radial drawing of $G$ induces a partition of its vertices into levels such that vertices in the same level are co-circular in the drawing; for each level, the planarity of the drawing induces a circular ordering of the vertices in the level. Conversely, in order to construct a radial drawing of $G$ a partition of its vertices into levels and a circular ordering within each level must be found such that vertices of the same level are drawn co-circularly and the edges can be drawn without intersecting each other.

Bachmaier et al. [1,2] investigate the radial planarity testing problem: Given a partition of the vertices of $G$ into levels, they want to test whether there exists a crossing-free radial drawing of $G$ consistent with the given leveling (i.e. vertices in the same level can be drawn on the same circle and the edges can be added without crossing). In [1] it is assumed that the edges are drawn as strictly monotone curves from inner to outer circles and that no two co-circular

\footnotetext{
* Research supported in part by "Progetto ALINWEB: Algoritmica per Internet e per il Web", MIUR Programmi di Ricerca Scientifica di Rilevante Interesse Nazionale, and by NSERC.
} 
vertices are connected by an edge. The elegant linear-time algorithm presented by Bachmaier et al. tests radial planarity by using an extension of $P Q$-trees, called $P Q R$-trees. In [2] the authors extend the algorithm to the case where edges between co-circular vertices are allowed.

In this paper we study radial drawings of planar graphs from a different perspective. We assume that the partition of the vertices of $G$ is not given and our goal is to compute a partition that minimizes the number of levels, i.e. that corresponds to a crossing-free straight-line radial drawing of $G$ on the minimum number of circles. We call such a drawing a minimum radial drawing of $G$. In contrast with the drawing conventions adopted in [1], we assume that the edges are straight-line segments and that vertices on the same level can be adjacent. These choices are justified by different application-oriented examples of radial drawings that adopt the straight-line standard (see e.g. $[12,13]$ ) and by the observation that allowing edges among co-circular vertices appears to be a natural approach for the reduction of the number of levels.

The contribution of the present paper is to characterize those graphs that can be drawn on a given number of concentric circles and to use this characterization to solve the above described optimization problem. More precisely:

- We show that every 2-outerplanar graph admits a crossing-free straightline radial drawing on two circles. The proof is constructive and the radial drawing can be computed in linear time. Preliminary results on computing radial drawing of 2-outerplanar graphs appear in [6].

- We generalize this results and characterize the family of graphs that admit a crossing-free straight-line radial drawing on at most $k \geq 2$ circles. We recall that similar characterization problems for straight-line $k$-layered drawings are studied for the case of $k \leq 3$; see, e.g. [5]. We also recall that a planar graph admits a drawing on one circle if each edge can bend at most once [7].

- Based on the characterization above, we show that there exists a polynomial time algorithm to compute a minimum radial drawing of a planar graph. The drawing has the additional property of being "proper", i.e. an edge always connects either co-circular vertices or vertices on consecutive circles.

For reasons of space some proofs are sketched or omitted.

\section{Preliminaries}

A 1-outerplanar embedded graph (also called 1-outerplane graph) is an embedded planar graph where all vertices are on the external face. An embedded graph is a $k$-outerplanar embedded graph (also called $k$-outerplane graph) $(k>1)$ if the embedded graph obtained by removing all vertices of the external face is a $(k-1)$-outerplane graph. The planar embedding of a $k$-outerplane graph is called a $k$-outerplanar embedding. A graph is $k$-outerplanar if it admits a $k$ outerplanar embedding. A planar graph $G$ has outerplanarity $k$ (for an integer $k>0$ ) if it is $k$-outerplanar and it is not $j$-outerplanar for $0<j<k$. In other words, the outerplanarity of $G$ denotes the minimum value of $k$ for which $G$ is $k$-outerplanar. 
Let $G$ be a $k$-outerplane graph with $k>1$. We associate a level with each vertex $v$ of $G$, denoted as $l e v(v)$, according to the following definition: $\operatorname{lev}(v)=0$ if $v$ is on the external face of $G$ and $\operatorname{lev}(v)=i(i=1, \ldots, k-1)$ if $v$ is on the external face after the removal of every vertex $u$ with $\operatorname{lev}(u)<i$. If $\operatorname{lev}(v)=i$, we say that $v$ is a vertex of level $i$. Let $V_{i}$ be the set of vertices $v$ with $\operatorname{lev}(v)=i$. The subgraph induced by $V_{i}$ is denoted by $G_{i}=\left(V_{i}, E_{i}\right)$. Notice that $G_{i}$ is a graph of outerplanarity 1 . Let $V_{i, i+1}=V_{i} \cup V_{i+1}$. The subgraph induced by $V_{i, i+1}$ is denoted by $G_{i, i+1}=\left(V_{i, i+1}, E_{i, i+1}\right)$.

We use $C_{0}, C_{1}, \ldots, C_{k-1}$ to denote a set of $k$ concentric circles in the plane, where the radius of $C_{i}$ is greater than the radius of $C_{i+1}(i=0, \ldots, k-2)$. Let $G$ be a planar graph and let $\Gamma$ be a crossing-free straight-line drawing of $G$. The drawing $\Gamma$ is a radial drawing if the vertices of $G$ are placed on a set of concentric circles. $\Gamma$ will be called a $k$-radial drawing of $G$ if it is a radial drawing on $C_{0}, C_{1}, \ldots, C_{k-1} . \Gamma$ is a minimum radial drawing if it uses the minimum number of circles. An edge $(u, v)$ with $u$ and $v$ on $C_{i}$ is called an intra-level edge. An edge $(u, v)$ with $u$ and $v$ on on $C_{i}$ and $C_{j}$ with $i \neq j$ is called an inter-level edge. If all inter-level edges of a radial drawing $\Gamma$ connect vertices on consecutive circles, $\Gamma$ is called a proper radial drawing.

Let $G$ be a $k$-outerplane graph. A radial drawing of $G$ is level-preserving if it is a $k$-radial drawing and every vertex $v$ with $l e v(v)=i$ is drawn on circle $C_{i}$. A level-preserving $k$-radial drawing of a $k$-outerplane graph is proper.

\section{Overview of the Approach}

We study the problem of computing a radial drawing of a planar graph $G$ on the minimum number of circles. We show that a minimum radial drawing of $G$ can be computed in polynomial time. Namely: (a) We prove that if a graph has outerplanarity $k$ then it admits a $k$-radial drawing; Also if a graph has a radial drawing on $k$-circles then it has outerplanarity at most $k$. (b) We use the above characterization and a result by Bienstock and Monma [3] to show that there exists an $O\left(n^{5} \log n\right)$-time algorithm that computes a minimum radial drawing of $G$.

The trickiest part is to show that a graph with outerplanarity $k$ has a $k$-radial drawing. We provide a linear-time algorithm that receives as input a $k$-outerplane graph $G$ and computes a level-preserving $k$-radial drawing of $G$. Our approach can be summarised as follows. We start with $G_{0}$, draw the vertices in $V_{0}$ on $C_{0}$ while maintaining their circular ordering in $G_{0}$. After placing $V_{i}$ on $C_{i}$ we compute the radius of $C_{i+1}$ and draw $V_{i+1}$ on $C_{i+1}$ without moving any vertex from $V_{j}$ with $0 \leq j \leq i$. For ease of presentation, we will define canonical $k$ outerplanar graphs and show how each $k$-outerplane graph can be transformed into a canonical $k$-outerplane graph. We will also show that a $k$-outerplane graph has a $k$-radial drawing if and only if its canonical form has a $k$-radial drawing.

\section{Canonical Graphs and Equipped $B C$-Trees}

Let $G$ be a $k$-outerplane graph. A mixed face of $G$ is a face containing vertices of two consecutive levels. $G$ is called inter-triangulated if all its mixed faces are 
three-cycles. Assume $G$ is inter-triangulated. Let $c$ be a cut-vertex of $G_{i+1}$. Let $B$ and $B^{\prime}$ be two blocks (i.e. biconnected components) of $G_{i+1}$ that are consecutive when going around $c$ in clockwise direction.

Since $G$ is inter-triangulated, there exists at least one edge of $E_{i, i+1}$ incident on $c$ that is encountered between $B$ and $B^{\prime}$ when going around $c$ in the clockwise direction. Such an edge of $E_{i, i+1}$ is called a separating edge because it separates blocks $B$ and $B^{\prime}$ around $c$. $G$ is said to be canonical if it is inter-triangulated and for any $i(i=0, \ldots, k-2)$ and for any two clockwise consecutive blocks $B, B^{\prime}$ of $G_{i+1}$ around a cut-vertex, there is exactly one separating edge.

Every connected $k$-outerplane graph can be made canonical as stated by the following lemma.

Lemma 1. Let $G$ be a connected $k$-outerplane graph with $n$ vertices. There exists an $O(n)$-time algorithm that computes an augmented graph $G^{\prime}$ such that: (1) $G^{\prime}$ is k-outerplane, (2) $G^{\prime}$ is canonical, and (3) the levels of the vertices of $G$ are preserved in $G^{\prime}$.

We now introduce equipped $B C$-trees. Let $G$ be a $k$-outerplane graph with $k>1$. We extend the block cut-vertex tree data structure [11] to identify specific subgraphs of $G$. Because of Lemma 1, we can (and will) restrict our attention to canonical graphs. Let $K$ be a connected component of $G_{i}$ with $i>1$. An equipped $B C$-tree $T$ of $K$ is an embedded rooted tree such that (for an illustration see Figures 1(a), 1(b), 1(c)):

- $T$ has three types of nodes: (a) A $B$-node for each block $B_{K}$ of $K$, referred to as the $B$-node of $B_{K}$. (b) A $C$-node for each cut-vertex $c$ of $K$, referred to as the $C$-node of $c$. (c) A $D$-node for each separating edge $e$ of $G$ that is incident on a cut-vertex of $K$, referred to as the $D$-node of $e$.

- If $K$ is biconnected, $T$ consist of a single $B$-node. If $K$ is not biconnected, we choose an arbitrary $C$-node as the root of $T$.

- The edges of $T$ are of two types: (a) Edges connecting a $C$-node of a cutvertex $c$ to a $B$-node of a block that contains $c$. (b) Edges connecting a $C$-node of a cut-vertex $c$ to a $D$-node of a separating edge incident on $c$.

- The planar embedding of $T$ reflects the embedding of $G$ : if $e$ is a separating edge incident on a cut-vertex $c$ and $e$ is between blocks $B$ and $B^{\prime}$ in clockwise ordering around $c$, then the $D$-node of $e$ is between the $B$-nodes of $B$ and $B^{\prime}$ in clockwise ordering around the $C$-node of $c$.

For example cut vertex 1 is chosen as the root of the tree shown in Figure 1(c). Separating edge $(2, b)$ in Figure $1(\mathrm{~b})$ separates blocks $A$ and $D$. Correspondingly, the $D$-node of $(2, b)$ appears between the $B$-nodes of $A$ and $D$ in the circular clockwise ordering around the $C$-node of cut-vertex 2 in the equipped $B C$-tree of Figure 1(c).

Let $\mu$ be the $C$-node of a cut-vertex $c$ of $K$, if $\mu$ is not the root of $T$, the parent of $\mu$ is a $B$-node and the leftmost child and the rightmost child of $\mu$ are $D$-nodes. If $\mu$ is the root of $T$ we arbitrarily choose the leftmost child of $\mu$ as a $D$-node; as a consequence the rightmost child of the root is a $B$-node. See for example Figure 1(c) where the rightmost child of the root is the $B$-node of $B$, while the leftmost child is the $D$-node of $(1, e)$. 


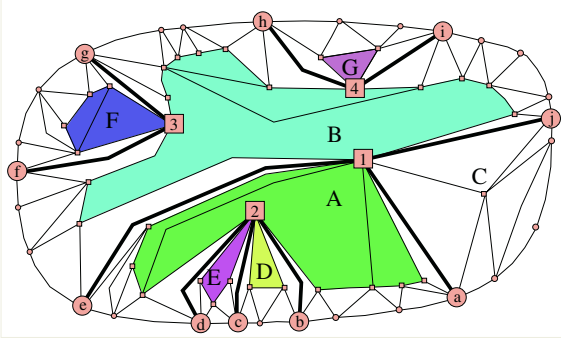

(a)

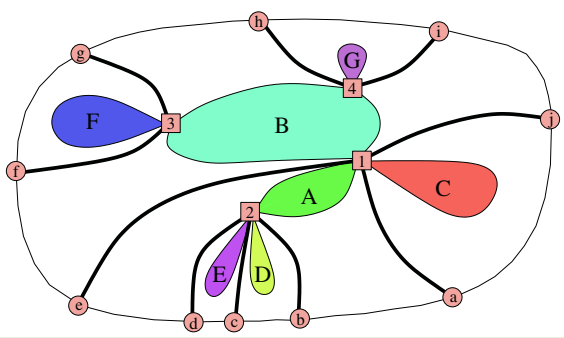

(b)

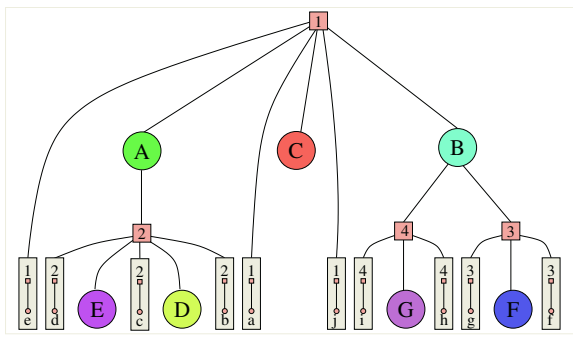

(c)

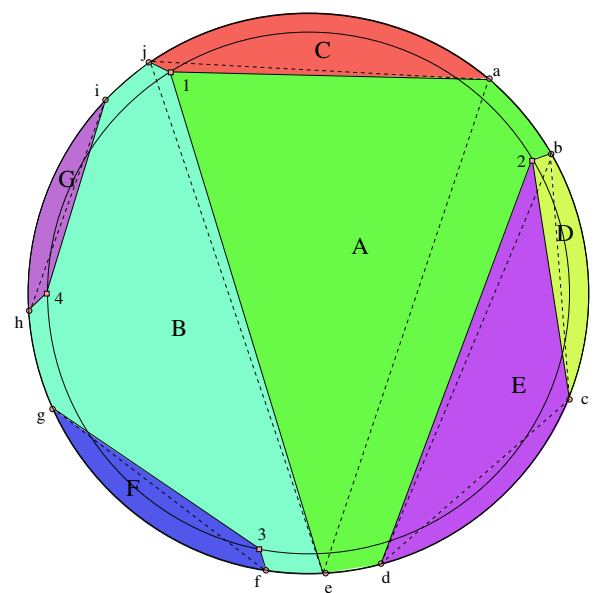

(d)

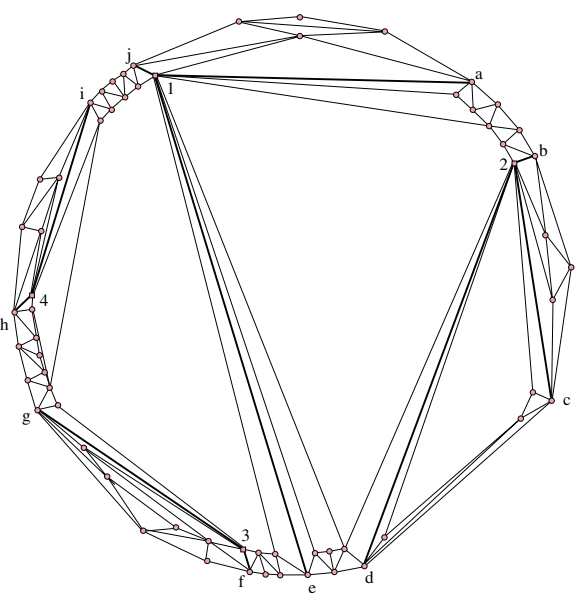

(e)

Fig. 1. (a) A 2-outerplane graph $G$. The blocks of $G_{1}$ are highlighted and labeled with capital letters. The cut-vertices of $G_{1}$ are numbered squares, and their separating edges are bold. (b) A schematic representation of the structure of $G$. The skeleton is highlighted with thick edges. (c) An equipped $B C$-tree of $G$ rooted at the $C$-node of cut-vertex 1. (d) A drawing of the skeleton of $G$. The labels of the regions reflect those of the corresponding blocks. (e) A level-preserving 2-radial drawing of $G$. 
Let $\nu$ be a $C$-node of a cut-vertex $c$ of $K$ that is not the root of $T$. Let $(c, v)$ be a separating edge of $K$. Vertex $v$ is called a separating vertex of $\nu$. For example, in Figure 1(c), vertices $b, c, d$ are separating vertices of the $C$-node 2. Let $\nu_{l}$ and $\nu_{r}$ be the leftmost and the rightmost $D$-node children (also called $D$-children) of $\nu$, and let $\left(c, v_{l}\right)$ and $\left(c, v_{r}\right)$ be their associated separating edges; $v_{l}$ and $v_{r}$ are called the leftmost separating vertex and the rightmost separating vertex of $\nu$, respectively. For example, vertices $d$ and $b$ are the leftmost and rightmost separating vertices of the $C$-node 2 in Figure $1(\mathrm{c})$.

Let $\mu$ be a $B$-node whose parent is a node $\nu$ where $\nu$ is the $C$-node of cut vertex $c$. Let $\nu_{r}, \nu_{l}$ be the $D$-children of $\nu$ that precede and follow $\mu$ in the clockwise ordering around $\nu$. Let $e_{l}=\left(c, v_{l}\right)$ be the separating edge associated with the D-node $\nu_{l}$ and $e_{r}=\left(c, v_{r}\right)$ be the separating edge associated with the D-node $\nu_{r}$. Edges $e_{l}$ and $e_{r}$ are called the left separating edge and the right separating edge of $\mu$, respectively. Also, $v_{l}$ and $v_{r}$ are called the left separating vertex and the right separating vertex of $\mu$, respectively. For example, $(2, d)$ and $(2, c)$ are the left and right separating edges for the $B$-node $E$ in Figure $1(\mathrm{c})$. A separating edge (separating vertex) of $\mu$ is either its left or right separating edge (vertex). The following lemma can be proven by using standard techniques for $B C$-trees [10].

Lemma 2. Let $G$ be a canonical 2-outerplane graph with $n$ vertices such that the subgraph $G_{1}$ induced by the vertices of level 1 is connected. There exists a $O(n)$-time algorithm that computes an equipped $B C$-tree of $G_{1}$.

The equipped block-cut-vertex tree $T$ of $K$ is used by the drawing algorithm described in the next section to split $G$ into small subgraphs each of which is drawn independently of the others. Note that if $K$ is biconnected the equipped $B C$-tree of $K$ has only one node, which is a $B$-node. However, in order to simplify the description of the drawing algorithm in the following sections, we assume that $K$ has at least one cut-vertex. The assumption is not restrictive, since we can always find a triangular mixed face $f$ consisting of one vertex $c$ of $K$ and two vertices $u, w$ of $G_{0}$, attach to $c$ a new dummy vertex $v$ in $f$, and triangulate the face again by adding two dummy edges $(v, u),(v, w)$. Vertex $v$ will be temporary added to $V_{1}$ and removed at the end of the drawing algorithm. The augmented graph is still canonical 2-outerplane and $c$ is now a cut-vertex.

\section{Radial Drawings of 2-Outerplanar Graphs}

Let $G$ be a 2-outerplane graph. In this section we show how to compute a 2radial drawing of $G$. This result will be a basic building block for the drawing techniques and the characterization of Section 6 .

By Lemma 1 we can assume that $G$ is canonical. Also, from the observation at the end of the previous section, we may assume that each connected component of $G_{1}$ has at least one cut-vertex. Let $K$ be a connected component of $G_{1}$. The subgraph of $G$ consisting of the separating edges of $K$ is called the skeleton of $K$ and is denoted as skel $(K)$. The skeleton of $G$ is the union of all $\operatorname{skel}(K)$, for 
every connected component $K$ of $G_{1}$. We denote it by $\operatorname{skel}(G)$. For example in Figure 1(a) and 1(b) the bold edges highlight the skeleton of the graph.

In order to use the algorithm as the basic tool to compute a $k$-radial drawing of a graph with outerplanarity $k$ (see Section 3 ), we assume that it receives as input a drawing $\Gamma_{0}$ of $G_{0}$ on a circle $C_{0}$ and that it computes a drawing $\Gamma$ of $G$ without changing $\Gamma_{0}$, i.e. $\Gamma_{0} \subset \Gamma$. We do not put any restrictions on the drawing $\Gamma_{0}$; the only hypothesis is that it preserves the planar embedding of $G_{0}$. The algorithm consists of four main steps:

1. Choice of $C_{1}$ : The radius of circle $C_{1}$ is determined.

2. Drawing the Skeleton of $G$ : For each connected component $K$ of $G_{1}$, the drawing of skel $(K)$ is computed. Figure $1(\mathrm{~d})$ shows a drawing of the skeleton of the graph of Figure 1(a).

3. Associating Blocks with Regions: Let $\Sigma_{K}$ be the drawing of skel $(K)$. $\Sigma_{K}$ induces a set of connected regions in the plane; each region is bounded by $C_{0}$ and by two separating edges. Each block is associated with a region and it will be drawn inside its region. Let $B_{K}$ be a block of $K$, let $T$ be an equipped $B C$-tree of $K$, and let $\mu$ be the $B$-node of $B_{K}$ in $T$. Let $\left(c, v_{l}\right)$ and $\left(c, v_{r}\right)$ be the left and right separating edges of $\mu$, respectively; let $\overline{c v_{l}}, \overline{c v_{r}}$ be the segments representing the separating edges of $\mu$ in $\Sigma_{K}$. Block $B_{K}$ is associated with the region bounded by $\overline{c v_{l}}, \overline{c v_{r}}$ and by the arc of $C_{0}$ from $v_{r}$ to $v_{l}$ in clockwise direction. For example, let $\Sigma_{K}$ be the drawing of Figure $1(\mathrm{~d})$ and consider block $D$ of Figure $1(\mathrm{~b})$. Block $D$ is associated with the region bounded in Figure $1(\mathrm{~d})$ by segments $\overline{2 c}, \overline{2 b}$, and by the arc of $C_{0}$ from $b$ to $c$ in clockwise direction.

4. Drawing the Blocks of Each Connected Component: The drawing of $G$ is computed by defining the coordinates of the vertices of $G_{1}$ that are not cut-vertices. To do that, the algorithm draws each connected component $K$ of $G_{1}$ independently. For each block $B_{K}$ of $K$ it draws $B_{K}$ inside its corresponding region, as defined in the previous step. Figure 1(e) shows a level-preserving 2-radial drawing of the graph of Figure 1(a).

\subsection{Choice of $C_{1}$}

Let $K$ be a connected component of $G_{1}$. The radius $r_{1}$ of $C_{1}$ depends on the drawing of $G_{0}$. Radius $r_{1}$ is chosen so that when a drawing of $\operatorname{skel}(G)$ is computed the region associated with each block contains an arc of $C_{1}$. This will be useful when drawing the vertices of the blocks inside their associated regions.

Let $K_{0}, K_{1}, \ldots, K_{h}$ be the connected components of $G_{1}$ and let $T_{j}$ be the equipped $B C$-tree of $K_{j}(j=0, \ldots, h)$. For each $B$-node of $T_{j}$ with separating vertices $v_{l}, v_{r}$, compute the distance between the point representing $v_{l}$ and the point representing $v_{r}$ in $\Gamma_{0}$. Let $\delta_{j}$ be the minimum of these distances over all $B$-nodes of $T_{j}$ and let $\delta=\min \left\{\delta_{j}: j=0, \ldots, h\right\}$. We define the radius of $C_{1}$ to be such that $C_{1}$ intersects the chords of $C_{0}$ with length $\delta$. Computing the radius of $C_{1}$ can be performed in a time that is linear in the number of blocks of $G$, and therefore linear in the number of vertices of $G$, since the graph is planar. 


\subsection{Drawing the Skeleton of $G$}

In this section we assume that the root of the equipped $B C$-tree $T$ of $K$ has at least three $B$-children. The case when the root has only two $B$-children can be handled similarly. For reasons of space we do not discuss this case.

The algorithm computes a drawing $\Sigma_{K}$ of $\operatorname{skel}(K)$ such that $\Sigma_{K}$ is inside the polygon of $\Gamma_{0}$ representing the face of $G_{0}$ that contains $K$ in $G$. The drawing algorithm computes $\Sigma_{K}$ so that for each block $B_{K}$, the separating edges that define the region associated with $B_{K}$ form a convex angle inside this region (the angle is called corner of the region in the following). This invariant will be used in the next steps of the algorithm and is important to prove the planarity of the computed drawing of $G$. The algorithm performs a top-down left-to-right visit of $T$. When a $C$-node $\nu$ is visited, the associated cut-vertex $c$ is drawn on $C_{1}$ together with all its incident separating edges. To better describe this algorithm we need to introduce some more terminology.

Let $\Gamma$ be a 2-radial drawing of $G$ on two circles $C_{0}$ and $C_{1}$ and let $p$ be a point on $C_{1}$. A free arc of $p$ is a maximal arc of $C_{1}$ having $p$ as one end-point and containing neither vertices of $\Gamma$ nor crossings between an edge of $\Gamma$ and $C_{1}$. Point $p$ has always two free arcs, one moving from $p$ clockwise (the left free arc of $p$ ) and the other moving from $p$ counterclockwise (the right free arc of $p$ ). Given any circle $C$, and two points $a$ and $b$ on $C$, the $\operatorname{arc}$ of $C$ traversed when moving from $a$ to $b$ clockwise will be denoted as $\langle a, b\rangle$. Points $a$ and $b$ will be called the first point and the last point of the arc, respectively. Each point of the arc distinct from $a$ and $b$ will be referred to as an internal point of $\langle a, b\rangle$. Finally, let $q$ be a point outside $C$. A point $p$ of $C$ is visible from $q$ if the segment $\overline{p q}$ does not cross $C$. The set of points of $C$ that are visible from $q$ is an arc called the visible region of $q$ on $C$. Note that, the first and the last points of the visible region of $q$ on $C$ are the intersection points between $C$ and the straight lines through $q$ tangent to $C$. The algorithm distinguishes among two cases:

- Node $\nu$ Is the Root of $T$. Let $\mu_{1}, \ldots, \mu_{h}$ be the $B$-children of $\nu$ and let $\mathcal{P}$ be the polygon defined by their separating vertices. From the choice of $C_{1}$, every side of $\mathcal{P}$ crosses $C_{1}$ in two distinct points. This implies that $\mathcal{P}$ contains a set of arcs of $C_{1}$. Draw $c$ as a point of one of these arcs. See for example the cut-vertex 1 in Figure 1(d).

- Node $\nu$ Is Not the Root of T. Let $u_{l}$ and $u_{r}$ be the leftmost and the rightmost separating vertices of $\nu$, respectively. By the choice of $C_{1}$ (Subsection 5.1), segment $\overline{u_{l} u_{r}}$ crosses circle $C_{1}$ in two distinct points; let $p$ be the intersection point that is closer to $u_{r}$, and denote by $\gamma$ the intersection of the left free arc of $p$ and the visible region of $u_{r}$. Draw $c$ as a point of $\gamma$. It can be proven that any point in $\gamma$ guarantees that the corners of the regions of the $B$-children of $\nu$ are convex. However, in order to correctly complete the drawing of the blocks of $K$ without changing the drawing of the skeleton (Subsection 5.4), the algorithm may need to make $\gamma$ smaller for some cases. Details about how to reduce $\gamma$ are omitted. 
Lemma 3. Let $G$ be a 2-outerplane graph with $n$ vertices, let $G_{0} \subset G$ be the subgraph of level 0 , and let skel $(G)$ be the skeleton of $G$. Let $\Gamma_{0}$ be an embedding preserving 1-radial drawing of $G_{0}$. There exists an $O(n)$-time algorithm that computes an embedding preserving 2-radial drawing $\Gamma$ of $G_{0} \cup \operatorname{skel}(G)$ such that $\Gamma_{0} \subset \Gamma$. Also, $\Gamma$ is a level-preserving drawing.

\subsection{Associating Blocks with Regions}

Since $G$ is canonical, the interior of each face $f$ of $G_{0}$ contains exactly one connected component $K$ of $G_{1} . K$ is drawn inside the region $f$ of the plane and is denoted by $\mathcal{R}_{f}$. Note that $\mathcal{R}_{f}$ is defined by $\Gamma_{0}$. Let $T$ be the $B C$-tree of $K ; \mathcal{R}_{f}$ is recursively subdivided into connected sub-regions $\mathcal{R}_{\mu}$, one for each $B$-node $\mu$ of $T$. As explained in Subsection 5.4, block $\mu$ is drawn inside $\mathcal{R}_{\mu}$. We formally define the regions induced by $\Sigma_{K}$ in the following.

For each $B$-node $\mu$ of $T$ let $\left(c, v_{l}\right)$ and $\left(c, v_{r}\right)$ be the separating edges of $\mu$. Denote by $\mathcal{R}_{\mu}^{*}$ the region of the plane delimited by the segments $\overline{c v_{l}}, \overline{c v_{r}}$, and by the arc $\left\langle v_{r}, v_{l}>\right.$ of $C_{0}$. The drawing technique of Subsection 5.4 will draw the blocks of the subtree of $T$ rooted at $\mu$ inside $\mathcal{R}_{\mu}^{*}$. The region $\mathcal{R}_{\mu}$ containing the single block associated with $\mu$ is as follows: (a) If $\mu$ is a leaf, let $\mathcal{R}_{\mu}=\mathcal{R}_{\mu}^{*}$. (b) If $\mu$ is an internal node with grandchildren $\mu_{1}, \ldots, \mu_{h}$, let $\mathcal{R}_{\mu}=\mathcal{R}_{\mu}^{*} \backslash\left(\mathcal{R}_{\mu_{1}}^{*} \cup \cdots \cup \mathcal{R}_{\mu_{h}}^{*}\right)$.

\subsection{Drawing the Blocks}

Let $B_{K}$ be a block of a connected component $K$ of $G_{1}$, and let $\mu$ be the $B$-node of $T$ representing $B_{K}$. As in Section 5.2, we assume in the following that the root of $T$ has at least three $B$-children.

A vertex of $G_{1}$ is an internal joint vertex if it is adjacent to at least two vertices of $G_{0}$. A vertex $u$ of $G_{0}$ is an external joint vertex if it is adjacent to at least two vertices of $G_{1}$. The algorithm that draws $B_{K}$ inside its associated region $\mathcal{R}_{\mu}$ distinguishes among two cases:

Case 1: $\boldsymbol{\mu}$ Is a Leaf of $\boldsymbol{T}$. Let $c$ be the cut-vertex associated with the parent of $\mu$ and let $c=a_{0}, a_{1}, \ldots, a_{t}$ be the internal joint vertices of $B_{K}$ in the clockwise order they appear on the external face of $B_{K}$. Since $G$ is inter-triangulated then the internal joint vertices $a_{l}$ and $a_{l+1}(l=0, \ldots, t-1)$ are adjacent to an external joint vertex, which is denoted by $u_{l+1}$. Also $a_{t}$ and $a_{0}$ are adjacent to an external joint vertex, which is denoted as $u_{t+1}$. Since $G$ is canonical, edges $\left(c, u_{1}\right)$ and $\left(c, u_{t+1}\right)$ are the separating edges of $\mu$.

The algorithm first places the internal joint vertices $a_{0}, \ldots, a_{t}$ in this order. At step $l(l=1, \ldots, t)$ vertex $a_{l}$ and its incident edges are added to the drawing. Each vertex $a_{l}$ is placed on $C_{1}$ as follows. If edge $\left(u_{l}, a_{l-1}\right)$ crosses $C_{1}$ then let $p$ be its crossing, otherwise let $p$ be coincident with $a_{l-1}$. Vertex $a_{l}$ is drawn as an internal point of the intersection between the right free arc of $p$ and the visible region of $u_{l}$.

Once all the internal joint vertices are placed, the algorithm draws the remaining vertices of $B_{K}$ and their incident edges. More precisely, let $v_{1}, \ldots, v_{r_{l}}$ 
be the vertices that are between vertices $a_{l}$ and $a_{l+1}(l=0, \ldots, t-1)$ in clockwise ordering on the external face of $B_{K}$. All these vertices are adjacent to the external joint vertex $u_{l+1}$. If edge $\left(a_{l}, u_{l+1}\right)$ crosses $C_{1}$ then let $q_{1}$ be its crossing, otherwise let $q_{1}$ be coincident with $a_{l}$. Analogously, if edge $\left(a_{l+1}, u_{l+1}\right)$ crosses $C_{1}$ then let $q_{2}$ be its crossing, otherwise let $q_{2}$ be coincident with $a_{l+1}$. Vertices $v_{1}, \ldots, v_{r_{l}}$ are drawn in this order as points of the arc $<q_{1}, q_{2}>$.

Case 2: $\boldsymbol{\mu}$ Is an Internal Node of $\boldsymbol{T}$. This case can be handled with techniques similar to those used for the previous case. We omit the decription of them for reasons of space.

Theorem 1. Let $G$ be a a 2-outerplane graph with $n$ vertices. $G$ admits a levelpreserving 2-radial drawing that preserves the embedding of $G$. Also there exists an $O(n)$-time algorithm that computes such a drawing.

\section{Minimum Radial Drawings of Planar Graphs}

In this section we first characterize the family of graphs that admit a radial drawing on at most $k$-concentric circles and then use the characterization to solve in polynomial time the problem of computing a minimum radial drawing of a planar graph.

Theorem 2. Let $G$ be a graph with outerplanarity $k$ and $n$ vertices. Then $G$ admits a proper $k$-radial drawing. Also, there exists an $O(n)$-time algorithm that computes such a drawing.

Proof. Since $G$ has outerplanarity $k$ then it has a $k$-outerplanar embedding. We show how to compute a level-preserving $k$-radial drawing $\Gamma$ of $G$ that preserves this embedding. This implies that $\Gamma$ is proper. An algorithm to compute $\Gamma$ is based on first drawing the subgraph induced by the vertices of level 0 on a circle $C_{0}$ and then by adding at each step the vertices of level $i$ on a circle $C_{i}$ $(i=1, \cdots k-1)$. At Step $i$ the subgraph $G_{i-1, i}$ is drawn by using the algorithm described in Section 5. Since $G=\bigcup_{i=0}^{k-2} G_{i, i+1}$ the computed drawing is a radial drawing of $G$. The fact that no two edges cross is a consequence of Theorem 1 . It follows that the above described algorithm computes a level-preserving $k$-radial drawing of $G$. As for the time complexity, it follows from Theorem 1 that the computation of drawing $G_{i-1, i}$ requires $O\left(n_{i}\right)$ time where $n_{i}$ is the number of vertices in $G_{i-1, i}$. Therefore the overall time complexity is $O(n)$.

Lemma 4. If a graph $G$ admits a $k$-radial drawing then it has outerplanarity at most $k$.

Proof. A $k$-radial drawing $\Gamma$ of $G$ defines an embedding of $G$. All vertices on the outerface of this embedding are drawn on $C_{0}$. Removal of all vertices on $C_{0}$ results in a $(k-1)$-radial drawing. So we can use induction to show that $\Gamma$ is a k-outerplane graph. It follows that the outerplanarity of $G$ is at most $k$.

Theorem 3. Let $G$ be a planar graph. $G$ admits a radial drawing on at most $k$-circles if and only if the outerplanarity of $G$ is at most $k$. 
Proof. Assume that $G$ has a $k$-radial drawing. Then by Lemma $4 G$ has outerplanarity at most $k$. Conversely, if $G$ has outerplanarity $j \leq k$, by Theorem 2 it admits a $j$-radial drawing with $j \leq k$.

Theorem 4. Let $G$ be a planar graph with $n$ vertices. There exists an $O\left(n^{5} \log n\right)$-time algorithm that computes a radial drawing of $G$ on the minimum number of concentric circles. Furthermore the computed drawing is proper.

Proof. Bienstock and Monma [3] describe an algorithm to compute the outerplanarity $k$ of $G$ and to determine a $k$-outerplanar embedding of $G$. This algorithm takes $O\left(n^{5} \log n\right)$ time. The result in [3] together with Theorem 3 imply that $k$ is the minimum number of circles for which there exists a radial drawing of $G$. The fact that such a drawing is proper is a consequence of Theorem 2. Again by Theorem 2 it follows that the time complexity of the whole algorithm is dominated by the technique in [3].

\section{References}

1. C. Bachmaier, F. Brandenburg, and M. Forster. Radial level planarity testing and embedding in linear time. In Proc. GD'03, volume 2912 of LNCS, pages 393-405, 2003.

2. C. Bachmaier, F. Brandenburg, and M. Forster. Track planarity testing and embedding. In Proc. SOFSEM'04, volume 2, pages 3-17, 2004.

3. D. Bienstock and C. L. Monma. On the complexity of embedding planar graphs to minimize certain distance measures. Algorithmica, 5(1):93-109, 1990.

4. S. Bornholdt and H. Schuster, editors. Handbook of Graphs and Networks: From the Genome to the Internet. Wiley-VCH, 2003.

5. S. Cornelsen, T. Schank, and D. Wagner. Drawing graphs on two and three lines. In Proc. GD'02, volume 2528 of LNCS, pages 31-41, 2002.

6. E. Di Giacomo and W. Didimo. Straight-line drawings of 2-outerplanar graphs on two curves. In Proc. GD'03, volume 2912 of $L N C S$, pages 419-424, 2003.

7. E. Di Giacomo, W. Didimo, G. Liotta, and S. K. Wismath. Curve-constrained drawings of planar graphs. Comp. Geometry: Theory and Appl. to appear.

8. M. Dodge and R. Kitchin. Atlas of Cyberspace. Addison Wesley, 2001.

9. S. N. Dorogstev and J. F. F. Mendes. Evolution of Networks, From Biological Nets to the Internet and $W W W$. Oxford University Press, 2003.

10. F. Harary. Graph Theory. Addison-Wesley, 1972.

11. F. Harary and G. Prins. The block-cutpoint-tree of a graph. Publ. Math Debrecen, 13:103-107, 1966.

12. M. Jünger and P. Mutzel, editors. Graph Drawing Software. Springer-Verlag, 2003.

13. K. Sugiyama. Graph Drawing and Applications for Software and Knowldege Engineers. World Scientific, 2002. 